\title{
Antenna Spatial Structure Influence on Communication System Capacity in Consideration of Transmitting Antenna Rotation
}

\author{
Yuri Parshin ${ }^{1}$, Sergey Gusev ${ }^{1}$ and Sergey Kolesnikov ${ }^{1 \text { a }}$ \\ ${ }^{1}$ Ryazan State Radio Engineering University, Department of Radio Engineering Devices, 390095 Ryazan, Russia
}

\begin{abstract}
Questions of technical implementation of communication system with different antenna spatial structures are discussed. The paper reviews the design of a system for data transmission from a rotating object to the ground receiving station with various spatial MIMO antenna structures. Different variants of the onboard antenna array with a different number of antennas are investigated. Values of the channel matrix for the communication system for the object rotating around the longitudinal axis are calculated. Transmission rate averaged over the period of rotation according to the position of the object on the trajectory and spatial structure of the ground receiving station is calculated.
\end{abstract}

\section{Introduction}

Design of high-speed data transmission system to a ground receiving station from rotating object requires solution of various research problems [1,2]. A feature of functioning conditions for information transmission system for some types of research equipment cargo is the rapid rotation of the object around the longitudinal axis specially implemented in order to stabilize the object when moving all over the path or just unwanted and uncontrolled rotation due to piloting malfunction. Also, when moving object changes orientation relative to ground reception point. All of this complicates the work of the information transmission system, requires matching directivities of onboard and ground-based antenna systems with the character of object motion, accounting of influence of changes in their relative orientation on the information transmission channel capacity.

The paper [2] outlines the problems associated with varying orientation of the object relative to ground reception point, which leads to lower data transmission efficiency. During object rotation the antenna blockage effect with object body may cause the interruption of information transmission. To improve the reliability of operation multiple ground reception points are used, and it leads to significant costs.

In this paper, in order to improve the quality of information transmission data MIMO technology [3], which has received widespread in mobile communication systems, is used. A special feature of the problem is the presence of multiple antennas on the rotating object to transmit information to a ground receiving station. Therefore, an actual problem is to optimize the transmitting part of the system that can be realized by space-time coding $[3,4]$.

The objective is to obtain capacity limit value in information transmission system from a rotating object with various spatial structures of antenna systems and recommendations on the technical implementation of the researched system.

\section{Problem Statement}

Let the object rotation axis be perpendicular to the plane of the receiving points. The rotation of the object is made with a certain angular velocity $\Omega$. Transmitting antennas are located on the object surface having form of a cylinder with radius $r$ uniformly along the circumference. Angular coordinates of antennas $\gamma_{T m}=2 \pi(m-1) / N_{T}, \quad m=1, \ldots, N_{T} \quad$ (Fig.1). The receiving antennas are located in the plane of object rotation by arc of radius $R$ uniformly in angular sector $\Delta \gamma_{R}$, angular coordinates of antennas $\gamma_{R n}=\Delta \gamma_{R}(n-1) / N_{R}, n=1, \ldots, N_{R}$.

Directivity of transmission antennas is determined by the type of radiators, location on the cylinder surface, influence of currents spreading on the cylinder surface, as well as the mutual influence of the radiators $[2,5]$. In the simple case, it can be assumed that the object body completely obscures the antenna and radiation pattern of transmitting antenna radiators in the plane of rotation is given by the expression

$$
D(\gamma)=\left\{\begin{array}{cc}
\cos \gamma, & \cos \gamma>0 \\
0, & \cos \gamma<0
\end{array}\right.
$$

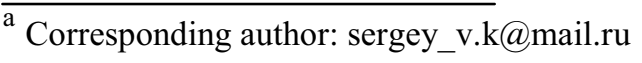


During object rotating the orientation of $\mathrm{m}$-th transmit antenna relative to $n$-th receiving antenna is defined by an antenna on the object and the receiving antenna on terrain

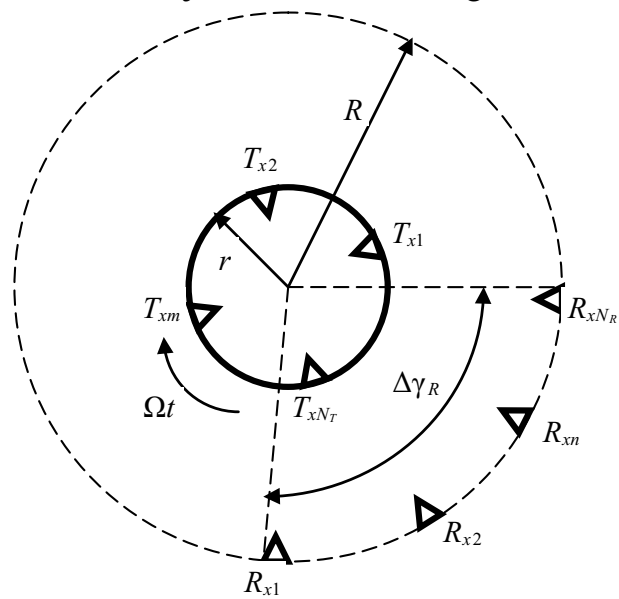

Figure 1. Spatial location of transmitting and receiving antennas

object slewing angle and angular positions of the transmitting respectively: $\gamma_{n m}=\Omega t+\gamma_{T m}-\gamma_{R n}$. Channel matrix coefficients are determined assuming direct line of sight between receiving and transmitting antennas and depend on the orientation of transmitting antennas in the direction of receiving antennas, and depend on the distance between the phase centers $R_{n m}$ of each pair of $m$-th transmitting and $n$-th receiving antennas [6]. Assuming that $R_{n m}>>r$ and variance of distance between antennas while rotating the object has almost no effect on the amplitude of received signal, we can write channel coefficient as:

$$
\underline{H}_{n m}=D\left(\gamma_{n m}\right) e^{-j \frac{2 \pi}{\lambda} R_{n m}}
$$

where

$$
\begin{aligned}
& R_{n m}=\left[\left(R \cos \gamma_{R n}-r \cos \left(\Omega t+\gamma_{T m}\right)\right)^{2}+\right. \\
& \left.+\left(R \sin \gamma_{R n}-r \sin \left(\Omega t+\gamma_{T m}\right)\right)^{2}\right]^{1 / 2}
\end{aligned}
$$
[3]:

The capacity of communication channel is given by

$$
C=\log _{2} \operatorname{det}\left(\mathbf{I}_{N_{R}}+\frac{q}{N_{T}} \underline{\mathbf{H}}^{H}\right)_{\mathrm{bit} / \text { symbol, }}
$$

where $\mathbf{I}_{N_{R}}$ - the unit diagonal matrix of dimension $N_{R}$, $q=D_{X} / D_{N}-$ system power budget, which is the ratio of signal power radiated from all transmitting antennas $D_{X}$ to the noise power at each receiving antenna $D_{N}$, $\underline{\mathbf{H}}=\left\{\underline{H}_{n m}, n=1, \ldots, N_{R}, m=1, \ldots, N_{T}\right\} \quad-\quad$ matrix of channel coefficients.

\section{Simulation Results}

Figures $2 \mathrm{a}, 2 \mathrm{~b}, 2 \mathrm{c}$ and $2 \mathrm{~d}$ show the dependence of channel capacity on the object rotation angle on the axis of rotation in a time equal to the period of object rotation at various number of receiving $N_{R}$ and transmitting antennas $N_{T}$. Receiving antennas are located in sector $\Delta \gamma_{R}=\pi / 4, R=10^{6} \lambda, r=5 \lambda$.

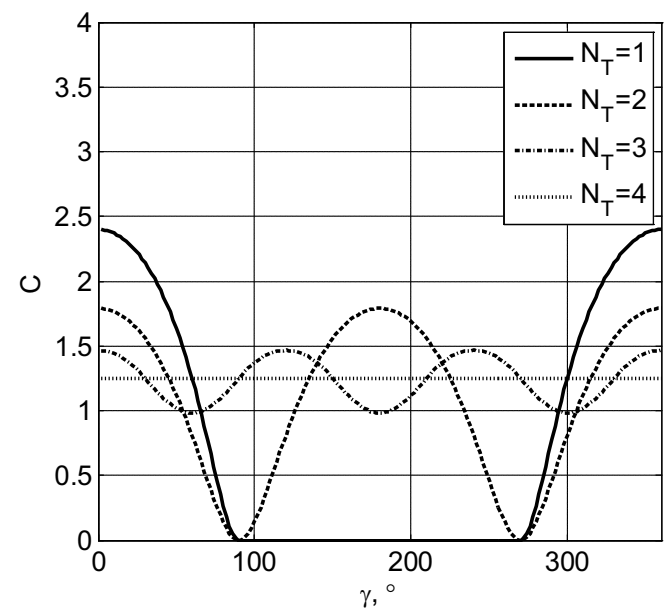

Figure 2a. Channel capacity vs object angular position for $N_{R}=1$

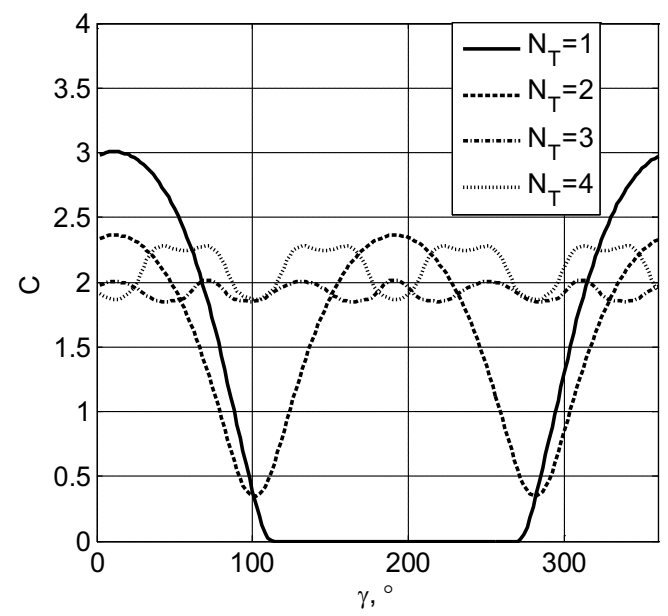

Figure 2b. Channel capacity vs object angular position for $N_{R}=2$

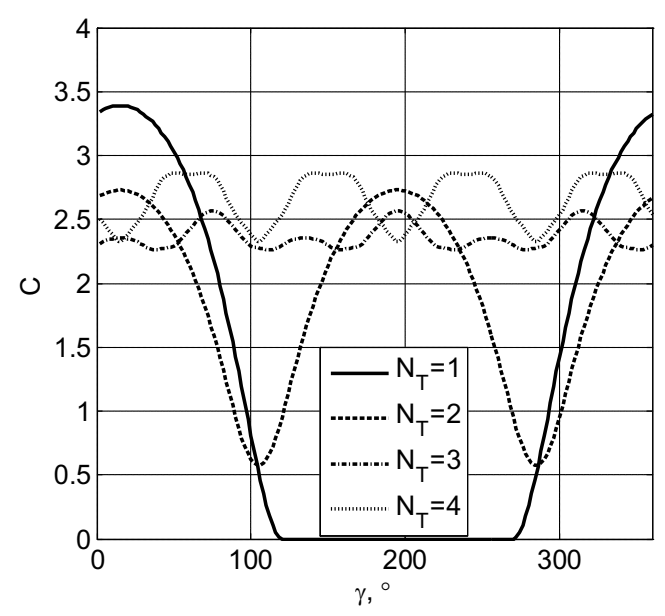

Figure 2c. Channel capacity vs object angular position $N_{R}=3$ 


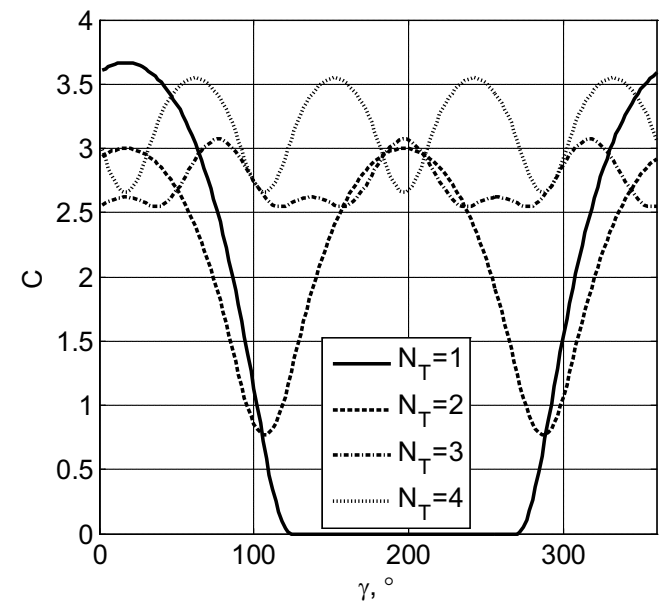

Figure 2d. Channel capacity vs object angular position for $N_{R}=4$

At high speed rotation of the object practical interest represents the average for the period of rotation information transmission channel capacity. In this case, the potential capacity may be obtained by using interleaving in a combination with efficient error correcting coding. Figures $3 \mathrm{a}, 3 \mathrm{~b}, 3 \mathrm{c}, 3 \mathrm{~d}, 3 \mathrm{e}$, and $3 \mathrm{f}$ show the dependence of average capacity on the angular position of the receiving antennas sector at different numbers of receiving $N_{R}$ and transmitting antennas $N_{T}$.

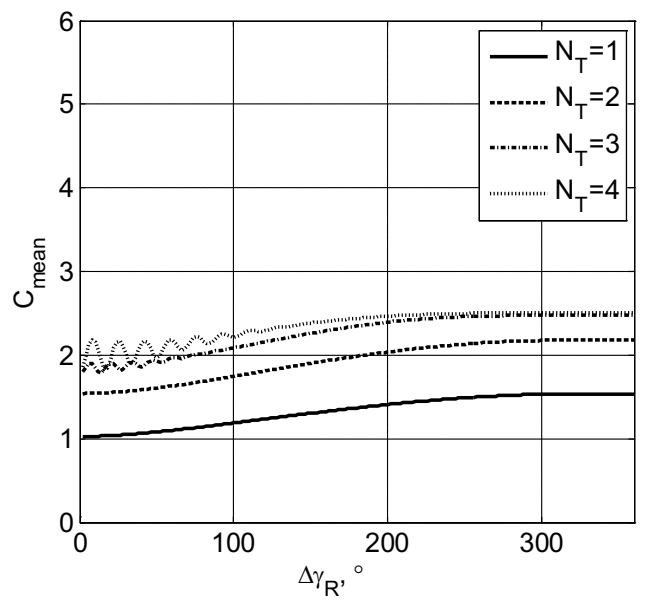

Figure 3a. Average channel capacity vs angular position of the receiving antennas for $N_{R}=2$

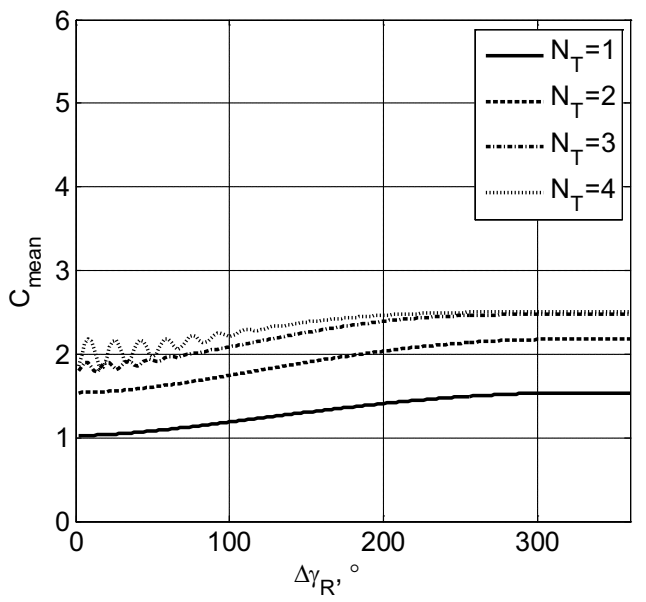

Figure 3b. Average channel capacity vs angular position of the receiving antennas for $N_{R}=3$

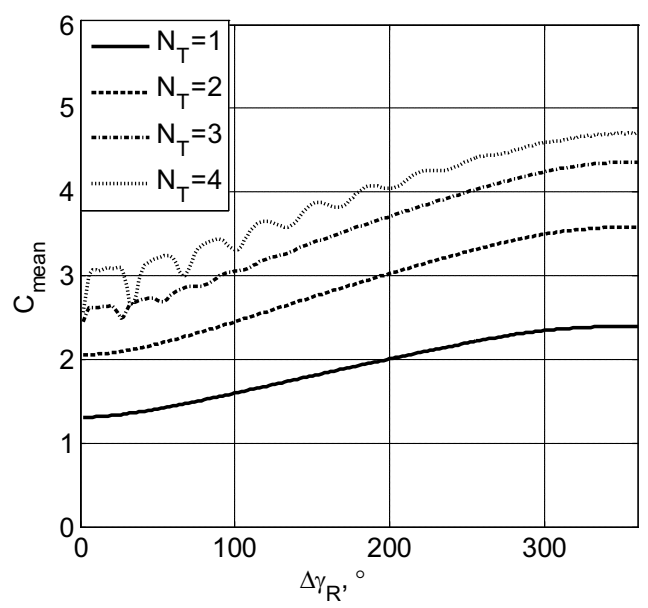

Figure 3c. Average channel capacity vs angular position of the receiving antennas for $N_{R}=4$

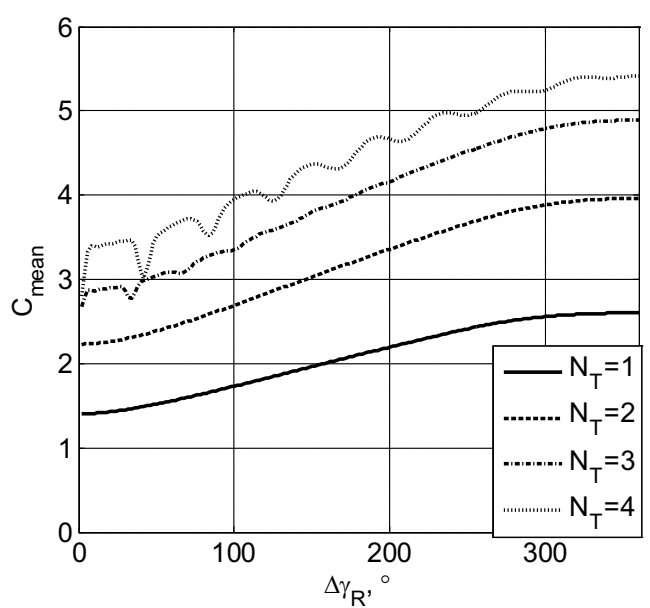

Figure 3d. Average channel capacity vs angular position of the receiving antennas for $N_{R}=5$ 


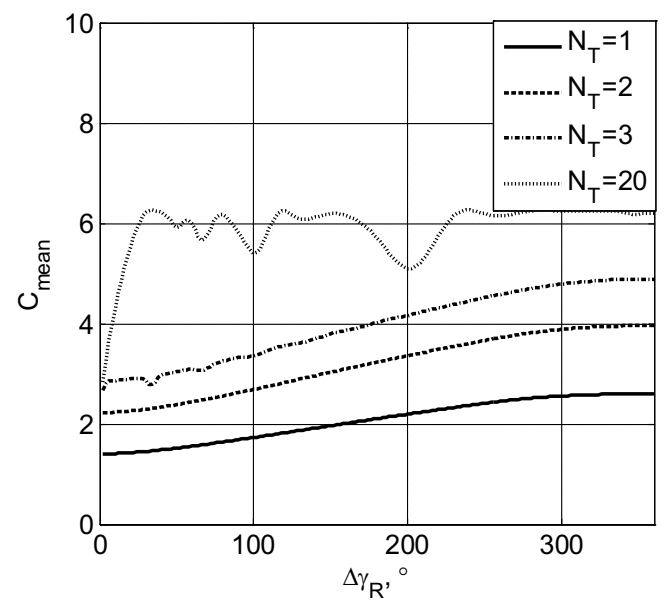

Figure 3e. Average channel capacity vs angular position of the receiving antennas for $N_{R}=5$

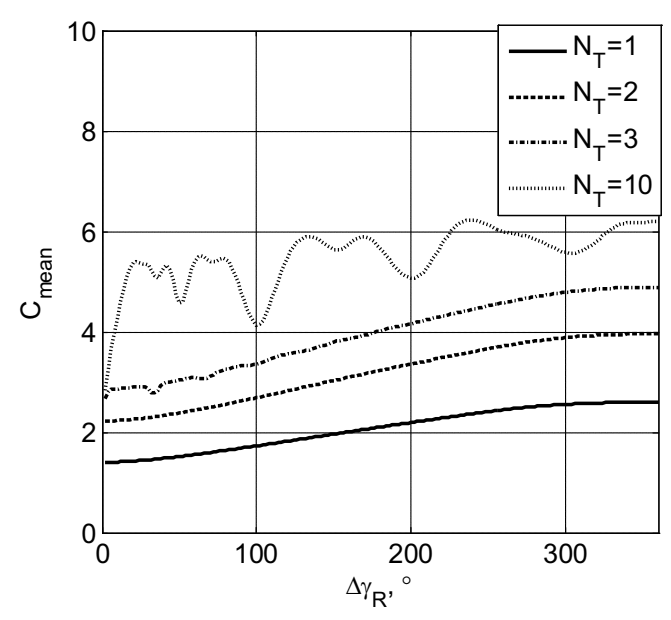

Figure 3f. Average channel capacity vs angular position of the receiving antennas for $N_{R}=5$

\section{Conclusion}

Based on the research of instantaneous and mean capacity depending on antenna spatial structure two modifications for technical implementation of high-speed data transmission system for a rotating object are advised.

In the first case it is assumed placement on board filled circular antenna array with antenna pattern, free from dips and diffraction lobes. In this case, minimum variation of instantaneous capacity depending on the object orientation and the maximum capacity is provided. At the same time limitations on mechanical strength of the object body and the complexity of implementing the transmission path, containing $N_{T}$ power amplifiers or microwave path for power distribution and phasing of antenna array elements from a single-channel transmitter does not allow to implement this variant with a large number of antennas. Besides, the implementation of multi-channel transmitter and microwave channel of phasing and power distribution with necessary efficiency is difficult because of limitations on the weight and size parameters of equipment.

The second variant of high-speed data transmission system assumes placing on object few-element circular antenna array and multiposition ground receiving system. The high mean capacity achieved using effective errorcorrecting coding. The realization of the encoding and decoding, which provides necessary data transmission rate may require significant computational resources. The main disadvantages of this method are extremely high requirements to memory volume of encoder, which is a part of transmitting equipment. The volume of required memory grows proportional to the square of object rotation period. The considered method is sensitive to width and number of deep dips of more than $10 \mathrm{~dB}$ in radiation pattern of onboard transmitting antenna array. In the presence of deep nulling the accuracy of transmitted information sharply reduced. An additional limitation of the second method is the necessity to measure coefficients of channel matrix with frequency depending on object speed and the requirement for the corresponding reverse link.

The research is carried out in the project of the Ministry of Education and Science of the Russian Federation №8.1697.2014/K

\section{References}

1. N. N. Bulgakov, V. G. Alybin ; A. A Krivoshein, Design features of the spacecraft vehicle onboard equipment of the command-measuring system to control it in the zone of its coverage from a ground station and outside it Microwave and Telecommunication Technology (CriMiCo), 2014 24th International Crimean Conference. Sevastopol, Crimea. - pp. 6-9.

2. E.P. Kolesnikov, Features of energy budget calculation of telemetry systems for missile launch, Radiotekhnika, 2004, №6, pp. 70-80.

3. Kuhn V. Wireless Communications over MIMO channels. Applications to CDMA and multiple antenna systems - John Wiley \& Sons, Ltd. 2006.

4. A.M. Shloma, M.G. Bakulin, V.B. Krejndelin, A.P. Shumov, Novye algoritmy formirovaniya i obrabotki signalov v sistemah podvizhnoj svyazi. Pod. red. prof. A.M. Shloma. Moscow: Goryachaya liniyaTelekom, 2008

5. G.T. Markov, D.M. Sazonov, Antenny: Uchebnik dlya studentov radiotekhnicheskih special'nostej VUZov. Moscow: Energiya, 1975.

6. Yu.N. Parshin, A.V. Ksendzov, "Effect of spatial correlation on the optimization efficiency of the spatial structure of multiple antenna system with receive diversity", Bulletin of Ryazan State Radio Engineering Academy, 2006, №19, pp. 54-62. 